\title{
TRIANGULAR TRUNCATION AND NORMAL LIMITS OF NILPOTENT OPERATORS
}

\author{
DON HADWIN
}

(Communicated by Palle E. T. Jorgensen)

\begin{abstract}
Dedicated to the memory of Domingo Herrero, who dazzled us with his brilliance, charmed us with his wit, and warmed us with his heart
\end{abstract}

\begin{abstract}
We show that, as $n \rightarrow \infty$, the product of the norm of the triangular truncation map on the $n \times n$ complex matrices with the distance from the normone hermitian $n \times n$ matrices to the nilpotents converges to $1 / 2$. We also include an elementary proof of $\mathrm{D}$. Herrero's characterization of the normal operators that are norm limits of nilpotents.
\end{abstract}

Suppose $n$ is a positive integer and let $\mathscr{M}_{n}, \mathscr{T}_{n}, \mathscr{N}_{n}$ denote, respectively, the sets of all $n \times n$ complex matrices, strictly upper triangular $n \times n$ matrices, and nilpotent $n \times n$ matrices. There is a natural mapping $\tau_{n}: \mathscr{M}_{n} \rightarrow \mathscr{T}_{n}$, namely, $\tau_{n}(T)$ replaces the entries on or below the main diagonal of $T$ with zeroes. The map $\tau_{n}$ is called triangular truncation on $\mathscr{M}_{n}$.

On an infinite-dimensional space, the triangular truncation mapping does not always yield the matrix of a bounded operator. This is related to the fact that the range of the mapping that sends a bounded harmonic function on the unit disk to its analytic part is not included in $H^{\infty}$. For example, if $f(z)=\log (1-z)$, then $u=2 i \operatorname{Im}(f)$ is bounded in modulus by $\pi$, but the analytic part of $u$, namely $f$, is not bounded. In terms of Toeplitz operators, $T_{u}$ is an operator with norm $\pi$, but the upper triangular truncation of $T_{u}$ is the formal matrix for $T_{f}$, which is not a bounded operator. The matrix for $T_{u}$ is the matrix whose $(i, i)$-entry is 0 and $(i, j)$-entry is $1 /(j-i)$ for $1 \leq i \neq j<\infty$. For each positive integer $n$, let $T_{u, n}$ be the $n \times n$ upper-left-hand corner of $T_{u}$, i.e., the $(i, i)$-entry of $T_{u, n}$ is 0 , and the $(i, j)$-entry of $T_{u, n}$ is $1 /(j-i)$ for $1 \leq i \neq j \leq n$. It follows that $\left\|T_{u, n}\right\| \leq \pi$ for each $n$, and that $\left\|\tau_{n}\left(T_{u, n}\right)\right\| \rightarrow \infty$ as $n \rightarrow \infty$. Hence $\left\|\tau_{n}\right\| \rightarrow \infty$ as $n \rightarrow \infty$.

Much work has been done in determining $\left\|\tau_{n}\right\|$. S. Kwapien and A. Pelczynski [KP, pp. 45-48] proved in 1970 that $\left\|\tau_{n}\right\|=O(\log (n)), \mathrm{K}$. Davidson [D, p. 39] proved that $\frac{4}{5 \pi} \leq \liminf _{n \rightarrow \infty}\left\|\tau_{n}\right\| / \log (n)$, and, in 1993, J. R. Angelos, C. Cowen, and S. K. Narayan $[\mathrm{ACN}]$ proved that

$$
\lim _{n \rightarrow \infty}\left\|\tau_{n}\right\| / \log (n)=1 / \pi \text {. }
$$

Received by the editors September 22, 1993; this paper was presented at a miniconference in honor of Eric Nordgren's sixtieth birthday held at the University of New Hampshire in June, 1993.

1991 Mathematics Subject Classification. Primary 47A58, 47B15; Secondary 15A60. 
All three of the papers cited above made use of the matrices $T_{u, n}$. It was shown in [ACN] that $\left\|T_{u, n}\right\| \leq \pi$ and, for $n \geq 2,\left\|\tau_{n}\left(T_{u, n}\right)\right\| \geq \log (n)-1$, which implies that $\left\|\tau_{n}\right\| \geq \frac{\log (n)-1}{\pi}$.

For each positive integer $n$ we define a number $\delta_{n}$ that measures how closely nilpotent $n \times n$ matrices can approximate norm-one hermitian ones, namely,

$$
\delta_{n}=\inf \left\{\|T-N\|: T \in \mathscr{M}_{n}, N \in \mathscr{N}_{n}, T=T^{*},\|T\|=1\right\} .
$$

It follows from the work of D. Herrero [H1] that $\delta_{n} \rightarrow 0$ as $n \rightarrow \infty$, and it was shown in [AFHV, Corollary A1.12] that

$$
\delta_{n} \geq \frac{\log (2)}{(1.038) \log (2)+\log (n)} .
$$

It was also pointed out in $[\mathrm{AFHV}]$ that $\mathrm{W}$. Kahan $[\mathrm{K}]$ proved that

$$
\delta_{n} \leq \frac{\pi+\log (2)}{2 \log (n)} .
$$

In this note we show a direct connection between $\delta_{n}$ and $\tau_{n}$ and prove $\lim _{n \rightarrow \infty} \delta_{n}\left\|\tau_{n}\right\|=1 / 2$. We then show how the fact that $\delta_{n} \rightarrow 0$ can be used to give an elementary proof of D. Herrero's theorem [H1] (Theorem 2 below), characterizing normal limits of nilpotent operators.

Theorem 1. $\lim _{n \rightarrow \infty} \delta_{n}\left\|\tau_{n}\right\|=1 / 2$.

Proof. Let $N_{n}=\tau_{n}\left(T_{u, n}\right)$. Then

$$
\left\|N_{n}\right\| \geq \log (n)-1 \text { and }\left\|\operatorname{Im}\left(N_{n}\right)\right\|=\left\|-i T_{u, n} / 2\right\| \leq \pi / 2 .
$$

Hence, $\left\|\operatorname{Re}\left(N_{n}\right)\right\| \geq\left\|N_{n}\right\|-\left\|\operatorname{Im}\left(N_{n}\right)\right\| \geq \log (n)-1-\pi / 2$. Thus,

$$
\delta_{n} \leq\left\|\left(N_{n}-\operatorname{Re}\left(N_{n}\right)\right) /\right\| \operatorname{Re}\left(N_{n}\right)\|\| \leq \frac{\pi / 2}{\log (n)-1-\pi / 2} .
$$

Hence

$$
\limsup _{n \rightarrow \infty} \delta_{n}\left\|\tau_{n}\right\| \leq \limsup _{n \rightarrow \infty} \frac{\left\|\tau_{n}\right\|}{\log (n)} \frac{(\pi / 2) \log (n)}{\log (n)-1-\pi / 2}=\frac{1}{\pi} \frac{\pi}{2}=\frac{1}{2} .
$$

On the other hand, suppose $A \in \mathscr{M}_{n}, N \in \mathscr{N}_{n}, A=A^{*},\|A\|=1$, and $\|A-N\|=\delta_{n}$. Then $\|N\| \geq 1-\delta_{n},\|\operatorname{Im}(N)\|=\|\operatorname{Im}(A-N)\| \leq \delta_{n}$. Since $N \in \mathscr{N}_{n}$, we can assume, via unitary equivalence that $N \in \mathscr{T}_{n}$. Hence $\tau_{n}(\operatorname{Im}(N))=N / 2$.

Hence,

$$
\left\|\tau_{n}\right\| \geq\|N / 2\| /\|\operatorname{Im}(N)\| \geq \frac{\left(1-\delta_{n}\right) / 2}{\delta_{n}},
$$

which implies that $\liminf _{n \rightarrow \infty} \delta_{n}\left\|\tau_{n}\right\| \geq \liminf _{n \rightarrow \infty}\left(1-\delta_{n}\right) / 2=1 / 2$.

Corollary. $\lim _{n \rightarrow \infty} \delta_{n} \log (n)=\pi / 2$.

We now show that $\lim _{n \rightarrow \infty} \delta_{n}=0$ leads to an elementary proof of Herrero's theorem on normal limits of nilpotent operators. Here $\sigma(T)$ denotes the spectrum of $T$. 
Theorem 2 (Herrero [H1]). Suppose $T$ is a normal operator on a separable, infinite-dimensional Hilbert space, $\sigma(T)$ is connected, and $0 \in \sigma(T)$. Then $T$ is a norm-limit of nilpotent operators.

The converse of the above theorem is also true. If $T$ is any operator that is a limit of noninvertible operators, then $0 \in \sigma(T)$, since the set of invertible operators is open. The fact that a norm limit of nilpotent operators must have connected spectrum uses the Riesz-Dunford functional calculus, namely, the first result in D. Herrero's book [H2] on approximation.

Proposition 3 [H2, Theorem 1.1]. Suppose $A$ is an operator and $\sigma(A)$ is the disjoint union of compact subsets $\sigma_{0}$ and $\sigma_{1}$, with $\sigma_{1}$ nonempty. Suppose also that $\Omega$ is a bounded open set containing $\sigma_{1}$ such that the closure $\Omega^{-}$is disjoint from $\sigma_{0}$. If $B$ is any operator with $\|B-A\|<\inf \left\{\left\|(z-A)^{-1}\right\|^{-1}: z \in \partial \Omega\right\}$, then $\sigma(B) \cap \Omega \neq \varnothing$.

Proof of Theorem 2. From the fact that $\delta_{n} \rightarrow 0$, we know that there is a sequence $\left\{A_{n}\right\}$ of norm-one hermitian matrices and a sequence $\left\{N_{n}\right\}$ of nilpotent matrices such that $\left\|A_{n}-N_{n}\right\| \rightarrow 0$. It follows that $\left\|N_{n}\right\| \rightarrow 1$, and we conclude that $\left\|A_{n}^{2}-N_{n}^{2}\right\| \rightarrow 0$. Since $\left\|A_{n}^{2}\right\|=1$ and $N_{n}^{2}$ is nilpotent, we can assume without loss of generality that $0 \leq A_{n} \leq 1$.

We remarked above that a norm limit of nilpotent operators must have connected spectrum. The $A_{n}$ 's, being finite matrices, cannot have connected spectrum; however, their spectra must try to fill out the interval $[0,1]$ as $n \rightarrow \infty$.

Lemma 4. For each $t$ in $[0,1]$, $\operatorname{dist}\left(t, \sigma\left(A_{n}\right)\right) \leq\left\|A_{n}-N_{n}\right\|$.

Proof. Suppose $0<t<1$, and assume that $t \notin \sigma\left(A_{n}\right)$. If we apply Proposition 3 with $A=A_{n}, B=N_{n}$, and $\Omega$ the disk centered at 1 with radius $1-t$, then the fact that $\sigma\left(N_{n}\right) \cap \Omega=\varnothing$ and the fact that $\inf \left\{\left\|(z-A)^{-1}\right\|^{-1}: z \in\right.$ $\partial \Omega\}=\operatorname{dist}\left(t, \sigma\left(A_{n}\right)\right)$ implies the desired inequality.

We next show how the operator $M_{x}$, multiplication by the independent variable $x$, on the space $L^{2}[0,1]$ with Lebesgue measure is a norm limit of nilpotents. Later, we will modify the argument to handle the general case.

Lemma 5. The operator $M_{x}$ is a norm limit of nilpotent operators.

Proof. Let $0 \leq t_{n_{1}} \leq t_{n_{2}}<\cdots<t_{n_{k(n)}}=1$ be the distinct eigenvalues of $A_{n}$. Let $A_{n}^{(\infty)}, N_{n}^{(\infty)}$ denote, respectively, a direct sum of infinitely many copies of $A_{n}, N_{n}$. Define $f_{n}:[0,1] \rightarrow[0,1]$ to be the simple function taking the value $t_{n_{k}}$ on the interval $\left[t_{n_{k-1}}, t_{n_{k}}\right)$ (where $\left.t_{n 0}=0\right)$.

It follows from Lemma 4 that $\left\|x-f_{n}(x)\right\|_{\infty} \rightarrow 0$; whence $\left\|M_{f_{n}}-M_{x}\right\| \rightarrow 0$. However, $A_{n}^{(\infty)}$ and $M_{f_{n}}$ are both diagonalizable operators with eigenvalues $t_{n_{1}}, t_{n_{2}}, \ldots, t_{\left.n_{k(n)}\right)}$, each having infinite multiplicity. Thus there is a unitary operator $U_{n}$ such that $U_{n}^{*} A_{n}^{(\infty)} U_{n}=M_{f_{n}}$, for each $n \geq 1$. Hence

$$
\begin{aligned}
\left\|U_{n}^{*} N_{n}^{(\infty)} U_{n}-M_{x}\right\| \leq & \left\|U_{n}^{*}\left[N_{n}^{(\infty)}-A_{n}^{(\infty)}\right] U_{n}\right\| \\
& +\left\|U_{n}^{*} A_{n}^{(\infty)} U_{n}-M_{f_{n}}\right\|+\left\|M_{f_{n}}-M_{x}\right\| \\
= & \left\|N_{n}-A_{n}\right\|+\left\|M_{f_{n}}-M_{x}\right\| \rightarrow 0 \text { as } n \rightarrow \infty .
\end{aligned}
$$


Since $U_{n}^{*} N_{n}^{(\infty)} U_{n}$ is a nilpotent operator for every $n$, we have proved that $M_{x}$ is a norm limit of nilpotent operators.

Lemma 6. If $T$ is a normal operator, $0 \in \sigma(T)$, and $\sigma(T)$ is connected, then there is a sequence $\left\{p_{n}\right\}$ of polynomials vanishing at 0 and and a sequence $\left\{W_{n}\right\}$ of unitary operators with $\left\|W_{n}^{*} p_{n}\left(M_{x}\right) W_{n}-T\right\| \rightarrow 0$.

Proof. Suppose $\varepsilon>0$, and let $\Omega=\{z+w: z \in \sigma(T),|w|<e\}$. Then $\Omega$ is open and connected; whence $\Omega$ is path-connected. Since $\sigma(T)$ is compact, we can find a finite subset $\mathscr{F}=\left\{\lambda_{1}, \lambda_{2}, \ldots, \lambda_{m}\right\}$ of $\sigma(T)$ with $\lambda_{1}=0$ such that every point in $\sigma(T)$ is within $\varepsilon$ of some point in $\mathscr{F}$. It follows from the spectral theorem that there is a diagonalizable operator $D$ whose set of eigenvalues is $\mathscr{F}$, with each eigenvalue having infinite multiplicity, such that $\|T-D\|<\varepsilon$. Since $\Omega$ is path-connected, there is a continuous function $\psi:[0,1] \rightarrow \Omega$ such that $\psi(0)=0$ and the range of $\psi$ includes $\mathscr{F}$. Since $\psi$ is uniformly continuous on $[0,1]$, there is a positive integer $m$ such that $\|\psi-h\|_{\infty}<\varepsilon$ where $h$ is the simple function taking on the value $\psi(k / m)$ on the interval $[k / m,(k+1) / m)$, for $0 \leq k \leq m-1$. Hence

$$
\left\|\psi\left(M_{x}\right)-h\left(M_{x}\right)\right\|=\left\|M_{\psi}-M_{h}\right\|<\varepsilon .
$$

However, $M_{h}$ is a diagonalizable operator, with each eigenvalue having infinite multiplicity, and such that every point in $\mathscr{F}$ is within $\varepsilon$ of the set of eigenvalues $M_{h}$.

It follows that every eigenvalue of $D$ is within $\varepsilon$ of an eigenvalue of $M_{h}$, and that each eigenvalue of $M_{h}$ is within $2 \varepsilon$ of an eigenvalue of $D$. By rearranging eigenvalues, we can find a unitary operator $W$ such that $\left\|W^{*} M_{h} W-D\right\|<2 \varepsilon$. Hence $\left\|W^{*} \psi\left(M_{x}\right) W-D\right\|<3 \varepsilon$. Since, by the Weierstrass approximation theorem, $\psi$ is a uniform limit of polynomials on $[0,1]$ (that vanish at 0 since $\psi$ vanishes at 0 ), there is a polynomial $p$ with $p(0)=0$ such that $\|p-\psi\|_{\infty}<\varepsilon$. Hence,

$$
\begin{aligned}
\left\|W^{*} p\left(M_{x}\right) W-T\right\| \leq & \| W^{*}\left[p\left(M_{x}\right)-\psi\left(M_{x}\right] W \|\right. \\
& +\left\|W^{*} \psi\left(M_{x}\right) W-D\right\|+\|D-T\| \\
< & \varepsilon+3 \varepsilon+\varepsilon=5 \varepsilon
\end{aligned}
$$

This completes the proof of the lemma.

The proof of Theorem 2 is completed by noting that, since the set $\mathscr{N}$ of nilpotent operators is closed under unitary equivalence and under evaluation of polynomials that vanish at 0 , the same is true for the norm closure $\mathscr{N}^{-}$. Since $M_{x} \in \mathscr{N}^{-}$, it follows that, for every unitary operator $W$ and for every polynomial $p$ with $p(0)=0$, we have $W^{*} p\left(M_{x}\right) W \in \mathscr{N}^{-}$. The preceding lemma clearly implies that $\mathscr{N}^{-}$contains every normal operator whose spectrum is connected and contains 0 . This completes the proof of Theorem 2.

It follows from the proof of Lemma 1 that we could take

$$
N_{n}=\left[\pi \tau_{n}\left(T_{u, n}\right) / \log (n)\right]^{2} \text { and } A_{n}=\left[\operatorname{Re}\left(N_{n}\right)\right]^{2}
$$

in the proof of Theorem 2. This, modulo the problem of computing the eigenvectors and eigenvalues of $A_{n}$, gives a "concrete" construction of a sequence of nilpotents that converge in norm to $M_{x}$ on $L^{2}[0,1]$.

The description of the closure of the set of all nilpotent operators on a separable infinite-dimensional Hilbert space is a deep theorem of C. Apostol, 
C. Foias, and D. Voiculescu [AFV]. A formula for the distance from an operator to the set of nilpotent operators is contained in [AFHV, 12.7].

\section{ACKNOWLEDGMENT}

The author wishes to thank the National Science Foundation for its support while this research was undertaken.

\section{REFERENCES}

[ACN] J. R. Angelos, C. C. Cowen, and S. K. Narayan, Triangular truncation and finding the norm of a Hadamard multiplier, Linear Algebra Appl. 170 (1992), 117-135.

[AFHV] C. Apostol, L. Fialkow, D. Herrero, and D. Voiculescu, Approximation of Hilbert space operators, Vol. II, Pitman, Boston, 1984.

[AFV] C. Apostol, C. Foias, and D. Voiculescu, Norm limits of nilpotents, II, Rev. Roumaine Math. Pures Appl. 19 (1974), 549-577.

[D] K. Davidson, Nest algebras, Pitman Res. Notes Math. Ser., no. 191, Longman Sci. Tech., Harlow, 1988.

[H1] D. A. Herrero, Normal limits of nilpotents operators, Indiana Univ. Math. J. 23 (1974), 1097-1108.

[H2] — Approximation of Hilbert space operators. I, Research Notes in Math., vol. 72, Pitman, Boston, 1982.

[K] W. Kahan, Every $n \times n$ matrix $Z$ with real spectrum satisfies $\left\|Z-Z^{*}\right\| \leq\left\|Z+Z^{*}\right\|$ $\left\{\log _{2} n+0.038\right\}$, Proc. Amer. Math. Soc. 39 (1973), 235-241.

[KP] S. Kwapien and A. Pelczynski, The main triangle projection in matrix spaces and its application, Studia Math. 34 (1970), 43-68.

Department of Mathematics, University of New Hampshire, Durham, New Hampshire 03824

E-mail address: don@math.unh.edu 